\title{
Innovation in cancer therapeutics and regulatory perspectives
}

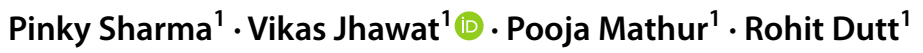 \\ Received: 18 November 2021 / Accepted: 3 February 2022 / Published online: 23 February 2022 \\ (c) The Author(s), under exclusive licence to Springer Science+Business Media, LLC, part of Springer Nature 2022
}

\begin{abstract}
Cancer therapy has undergone a drastic revolution in the past few decades with the introduction of several novel therapies, like immunotherapy (active and passive), stem cell-based therapies, and nanocarrier-based therapies. These therapies have addressed the issues of conventional cancer therapy (chemotherapy or radiotherapy), like specificity and off-target effects. Further, the introduction of such treatments has improved survival and converted a terminal disease into a more manageable condition. However, many clinical, ethical, and regulatory issues are raised with such novel additions. Several effective therapies are under research but could not come to market or are delayed due to regulatory concerns for marketing approval. The scope of this review encompasses the examination of these regulatory issues and discuss their possible solutions. A practical and flexible regulatory approach, harmonized globally, could help the patients suffering from a terminal illness to lead a quality life.
\end{abstract}

Keywords Cancer therapy $\cdot$ Novel therapy $\cdot$ Immunotherapy $\cdot$ Regulatory challenges

\section{Introduction}

Cancer spans the globe as one of the prime causes of mortality [1]. The cancer burden is rapidly increasing worldwide, as depicted by the GLOBOCON 2020 [2] estimates. These estimates, produced by International Agency for Research on Cancer, suggested 19.3 million new cases of all cancer types with 10 million cancer deaths in 2020. Further, it is estimated to rise to 28.4 million cases in 2040 (47\% increase) [1]. Additionally, the GLOBOCON 2020 [2] estimates (both sexes) suggested breast cancer to be the most frequently diagnosed cancer, followed by lung, colorectal, prostate $(7.3 \%)$, and stomach $(5.6 \%)$ cancer. In females, breast cancer was the prime cause of mortality, followed by lung and colorectal cancer. Whereas in males, the prime cause of mortality was lung cancer, followed by liver and colorectal cancer [1]. This high rate of cancer incidence and mortality is of concern for developing effective cancer treatment and prevention measures.

Figure 1 demonstrates the cancer incidence and projected cancer burden from 2018 to 2040 in India. It was observed

Vikas Jhawat

jhawat231287@gmail.com

1 School of Medical and Allied Sciences, GD Goenka

University, Gurugram, Haryana, India that the projected cancer burden in females is comparatively more than in men in the coming years [3].

Currently, cancer treatments include surgery, radiation therapy, chemotherapy, targeted therapy, hormonal therapy, and immunotherapy. Conventional cancer therapy included chemotherapy and radiotherapy, which had undergone a radical change after the introduction of targeted therapy [4]. The chemotherapeutic agents were the first revolutionary pharmacological approach for treating cancer as these agents were cytotoxic to the tumors [5]. They are generally designed to hit the de-regulated mechanisms inside the cancer cells [6]. Alkylating agents and antimetabolites were the major chemotherapeutic agents earlier. The alkylating agents (nitrogen mustard-based drugs) added alkyl groups onto bases in deoxyribonucleic acid (DNA), leading to cytotoxicity. Antimetabolites (aminopterin, methotrexate, mercaptopurine, fluorouracil, gemcitabine, etc.) halt DNA replication and thus inhibit cell proliferation. Besides these agents, drugs included in the chemotherapy were anti-mitotic agents (Vincristine, etoposide, paclitaxel, topotecan, taxol, etc.), cytotoxic antibiotics (doxorubicin, actinomycin D, bleomycin, etc.) and others, like polyamine transport inhibitors and iron-modulating agents (desferrioxamine) [5]. Since the 1960s, combination therapies came into existence, and consequently, patient outcomes have improved [7]. 
Fig. 1 Cancer incidence in Indian men and women in 2018. A Incidence rates are per 100,000 population grouped by age and sex. B Projected cancer burden by sex from 2018 to 2040. Data from WHO. Adapted from [3]
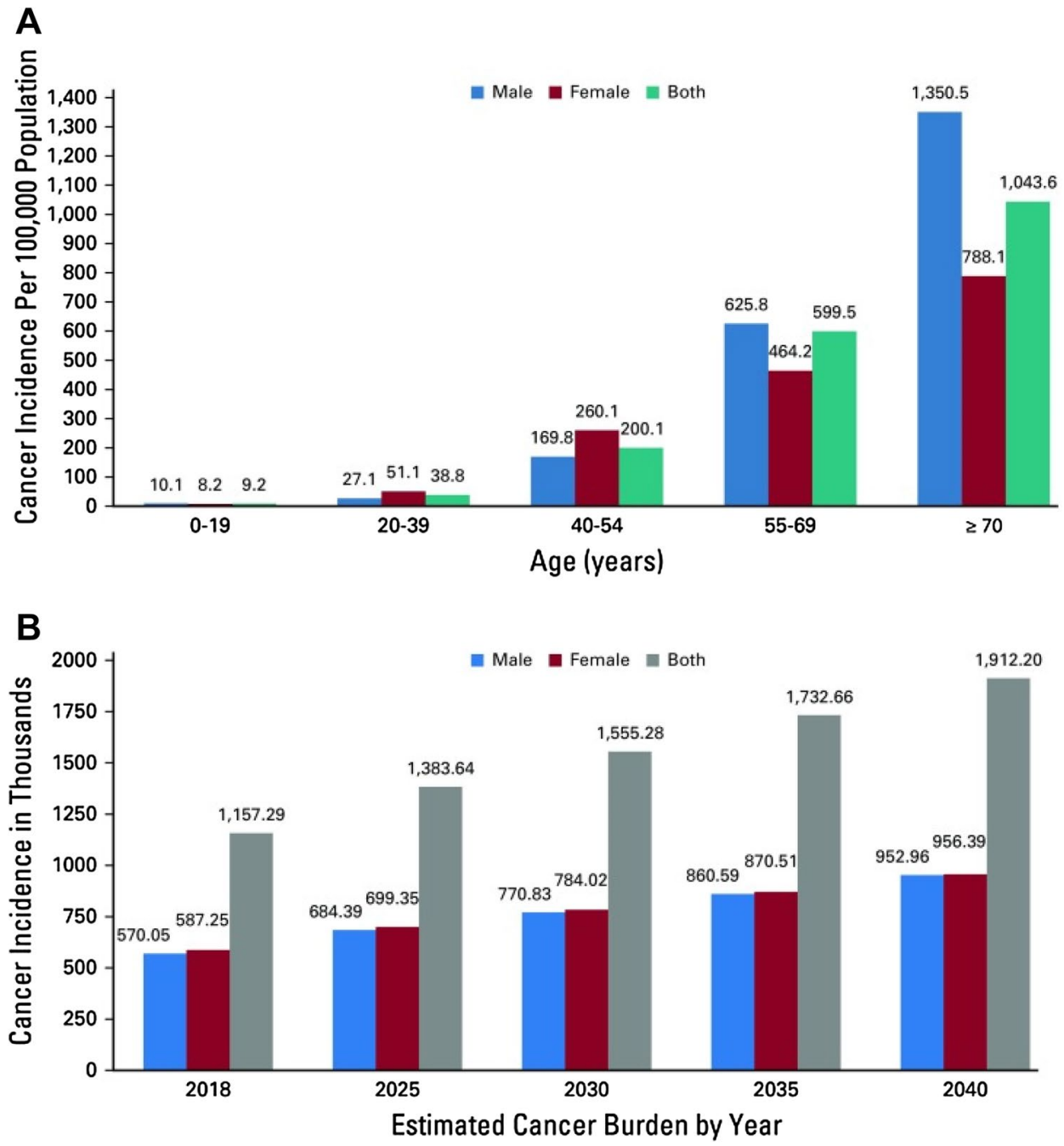

However, systemic chemotherapy is less specific to tumors and has prominent side effects [8] and thus, more tolerable and effective therapies are needed. Since the knowledge of tumor biology has expanded, cancer therapy has also changed paradigms in the last few decades. The two strategies that brought a revolution in cancer management were personalized therapy and targeting the tumor microenvironment with either small molecules or immunotherapy [9]. This review encompasses the intricacies of such novel therapies for cancer management and discusses the challenges faced in the development of such therapies.

\section{Novel cancer therapies}

Several novel and innovative ways of intervening in cancer progression have been developed and few are still under development. These novel therapies are comparatively well tolerated with better efficacy than the conventional chemotherapeutic agents. The novel therapies currently in clinical practice include hormonal therapies, targeted therapies, like immunotherapy, cancer vaccines, and stem cell-based therapy. The details of these therapies are discussed as below:

\section{Hormone therapy}

Hormonal therapy is beneficial for hormone-dependent cancers, such as breast, prostate, endometrial, and uterine cancers. This therapy either interferes with the hormone production or alters the receptor activity [10]. The hormonal therapy may be indicated as neoadjuvant, adjuvant to surgeries, or palliative therapy for metastatic cancers (breast or prostate) [11]. Several hormone analogs are employed in cancer therapy, which commonly include corticosteroids, somatostatin, and progestins. Besides, inhibitors of hormone synthesis (gonadotrophin-releasing hormone agonists or antagonists, aromatase inhibitors) and inhibitors of hormone receptors (selective estrogen receptor modulators, 
antiandrogens) are also widely used in breast and prostate cancers $[10,11]$.

\section{Cancer immunotherapy}

Targeted therapies, such as immunotherapy, precisely aim at the tumor site and spares the surrounding host cells. Hence, this therapy is more specific and comparatively lesser side effects [12]. Cancer immunotherapy mainly targets the patient's immune system and empowers it to attack and eradicate the cancerous cells. Several cancer immunotherapies have been proven to be effective in various cancers, like renal cancer, lung cancer, bladder cancer, breast cancer, melanoma, and Hodgkin's lymphoma [13]. Monoclonal antibodies (mAbs), immune checkpoint blockers, cytokines, cancer vaccines, and cell-based immunotherapy are effective approaches approved to date [14]. Many of these approaches target a specific protein narrowing their range of activity, while some can manipulate multiple targets with a broad range of activity. Further, in addition to widely available immunotherapeutic approaches (monoclonal antibodies), some approaches can also be personalized by applying genetic engineering [13].

\section{Monoclonal antibodies}

Monoclonal antibodies have now become an integral part of cancer therapy, like chemotherapy and radiation therapy. They are small recombinant proteins developed against specific targets, like receptors or cellular proteins [5]. These antibodies can work in multiple ways. They can use direct cytotoxic action on tumor cells or stimulate the immune system to develop anti-tumor responses [15]. Trastuzumab was the first $\mathrm{mAb}$ against human epidermal growth factor receptor 2 (HER2)/neu receptor of breast cancer being tested in a clinical trial. At the same time, rituximab targeting surface antigen CD-20 in B-cell lymphoma was the first approved $\mathrm{mAb}$ [5]. To date, there have been $30 \mathrm{mAbs}$ approved by FDA and/or EMA for cancer therapy $[16,17]$. These antibodies have been developed against targets, like HER2 (breast cancer), epidermal growth factor receptor (EGFR, colorectal, head, and neck cancer), and CD proteins (lymphoma, melanoma) [14].

\section{Anti-angiogenesis}

Additionally, mAbs have also been developed targeting neo-angiogenesis that explicitly alters the tumor microenvironment and reverts the immunosuppressive state into the immune-promoting state. Such a combination of immunotherapy with anti-angiogenesis enhances the retardation of the tumor. The major signaling pathways for promoting angiogenesis in tumors include vascular endothelial growth factor (VEGF), VEGF-receptor (VEGFR), platelet-derived growth factor (PDGF), PDGF receptor (PDGFR), fibroblast growth factor (FGF), and FGF receptor. Of these, the research on VEGF has been extensive, and several mAbs have been developed targeting angiogenesis through VEGF [18]. Bevacizumab was the first anti-angiogenic $\mathrm{mAb}$ directed against VEGF. It has been proven to be a very efficient drug for cancer treatments like non-small cell lung cancer along with first-line therapy for colorectal cancer [19]. However, angiogenesis is a complex multi-step process and involves combined actions of cytokines, extracellular matrix, and cell adhesion molecules. Thus, angiogenesis inhibitors also include multi-kinase inhibitors or serine-threonine kinase inhibitors in addition to the monoclonal antibodies [20]. These molecules are discussed later in the review.

\section{Immune checkpoint inhibitors}

The tumor microenvironment serves as a physical barrier to $\mathrm{T}$ cells. Besides, the tumor can also affect the T-cell generation and activation and produce angiogenic and immunosuppressive factors [prostaglandin, programmed death (PD-1), PD ligand (PD-L1), cytotoxic T-lymphocyte-associated antigen (CTLA)]. These mechanisms help the cancer cells to evade the immune response and escape the T-cell immunity. The immune checkpoint inhibitors are mAbs that target these immune-suppressive mechanisms of the tumor and prevent the immune escape of the cancer cells, providing passive immunity [5, 9, 21]. Monoclonal antibodies, anti-CTLA, anti-PD-1, or anti-PD-L1 have shown clinical benefits in some cancers (melanoma, non-small cell lung cancer, merkel cell carcinoma, urothelial carcinoma, or renal cancer) $[9,14]$. Ipilimumab was the first FDA-approved immune checkpoint inhibitor mAb directed toward CTLA-4 for the treatment of metastatic melanoma. Later, anti-PD-1 and antiPD-L1 drugs also received approval for other solid tumors (metastatic melanoma, non-small cell lung cancer, urothelial carcinoma, head and neck cancer, or renal cancer) [22]. Metastatic tumors were earlier difficult to be operated, as it was unlikely to provide any benefit. However, immune checkpoint inhibitors are helpful for such advanced cancer patients wherein they could reduce or eliminate the tumor to be surgically removed.

Further, the response observed with these agents was remarkable and durable [14]. The effectiveness of these agents has led to the further development of other targets, such as lymphocyte activation gene 3 (LAG3), T-cell immunoglobulin and mucin domain-containing 3 (TIM3), T-cell immunoglobulin and immunoreceptor tyrosine-based inhibitory motif domain (TIGIT), and V-domain immunoglobulin suppressor of T-cell activation (VISTA) [15, 22]. However, it is essential to note that the effectiveness of these agents 
was observed in only a minority of patients and thus, combination therapy using multiple targeted therapies may be developed [14].

\section{Cell-based immunotherapy}

Cell-based immunotherapy, also called adoptive T-cell therapy, involves transferring T cells (natural or genetically modified) into metastatic cancer patients. These $\mathrm{T}$ cells (allogeneic or autologous) are expanded ex vivo before the transfer in the patients to improve the specificity and antitumor activity $[13,21]$. This therapy has been proven extensively with the use of tumor-infiltrating lymphocytes. The lymphocytes were isolated from a cancer biopsy, expanded ex vivo, and then transferred to the same patient along with interleukins (IL-2). This therapy has produced durable effectiveness in patients with advanced metastatic melanoma [13, 22]. However, such lymphocytes would be able to target the tumor-specific antigens that are presented by the major histocompatibility complex (MHC) and not the surface antigens.

The chimeric antigen receptor $\mathrm{T}$ therapy (CAR-T), modular synthetic T-cell therapy, is one of the most successful autologous cell-based immunotherapies. The CAR-T cells can produce cytotoxicity, independent from MHC, by targeting the surface antigens as well. The $\mathrm{T}$ cells (allogeneic or autologous) are genetically engineered, expanded ex vivo, and then infused back in the patient [22]. The CAR-T-cell therapy has been developed targeting several surface antigens, like CD19, CD28, and CD40. It has shown unparalleled success in targeting CD19 in B-cell leukemia or lymphoma, for which FDA approval is also granted. Newer strategies are now attacking multiple tumor antigens by developing multi-target CAR constructs. These are experimented in solid tumors, like multiple melanomas, glioblastoma, and breast cancer [23]

\section{Cancer vaccines}

The cancer vaccines form the active immunotherapeutic products compared to the therapies discussed above (passive immunotherapy) [24]. They can be prophylactic or therapeutic. Prophylactic vaccines can reduce the occurrence of cancers like hepatocellular or cervical cancer due to oncogenic viruses. In contrast, therapeutic vaccines stimulate the immune system to destroy the neoplastic cells [22]. These vaccines comprise either part or the whole antigens present in the cancer cells to induce immunity [25].

The selection of the antigens for the development of vaccines has always been crucial as the antigen should be possessing the following characteristics: only expressed by the cancer cells, present on all cancer cells, essential for the survival of the cancer cells, and immunogenic. Several types of antigens have been tried to develop vaccines, like tumor-associated antigens (e.g., HER2, prostate-specific antigen), oncogenic viral antigens, or neoantigens (such as CTLA-4, PD-1). Besides, different vaccine vectors have been developed: cell-based vaccines (tumor cell-based, dendritic cell-based), virus-vector vaccines, and molecular vaccines (peptide-based or DNA/RNA-based). However, the overall results of using cancer vaccines have not been very encouraging and many of them are still under research. The first cancer vaccine to achieve FDA approval was sipuleucel$\mathrm{T}$ for metastatic prostate cancer $[13,14,25]$.

\section{Combination therapies}

Combination therapy is a significant pillar of cancer therapy. Combining two or more immunotherapies or immunotherapy with traditional chemotherapies can target multiple pathways in cancer progression and improve the overall survival or response rate in cancer patients [22, 26, 27]. Several combination therapies are being studied for their effectiveness in the survival and response rates in different types of cancer. These combinations include immune checkpoint inhibitors combined with other checkpoint inhibitors (e.g., anti-CTLA-4 with anti-PD-1/PD-L1 mAbs), checkpoint inhibitors (ipilimumab and nivolumab) with chemotherapy (carboplatin, paclitaxel, cisplatin) or radiotherapy, CAR-Tcell therapy with checkpoint inhibitors (e.g., pembrolizumab or nivolumab with CAR-T cell), and cancer vaccines with chemotherapy (e.g., peptide-based cancer vaccine for renal cell carcinoma with cyclophosphamide) [26]. FDA approval has been granted for ipilimumab and nivolumab combination therapy in metastatic melanoma and renal cell carcinoma [22]. Thus, combination therapies may be promising in the future for several other cancers as well.

\section{Small-molecule inhibitors}

Targeted drug therapy in cancer involves either macromolecules (e.g., mAbs) or small molecules. The small molecules encompass a wide range of targets, like kinases (tyrosine kinase, serine/threonine kinase), epigenetic mechanism, B-cell lymphoma (Bcl-2) proteins, hedgehog pathway, proteasomes, and poly ADP-ribose polymerases (PARPs). The kinase inhibitors may include tyrosine kinase receptor inhibitors [anaplastic lymphoma kinase (ALK) inhibitors, cellular-mesenchymal-epithelial transition factor (c-Met) inhibitor, epidermal growth factor receptor (EGFR) inhibitor, Fms-like tyrosine kinase 3 (FLT3) inhibitors, VEGFR/ FGFR/PDGFR inhibitors, tropomyosin receptor kinase [TRK] inhibitors), non-receptor tyrosine kinase inhibitors (Bcr-Abl1 receptor inhibitors, Bruton's agammaglobulinemia tyrosine kinase (BTK) inhibitors, Janus kinase (JAK) inhibitors], and serine/threonine kinase inhibitors [BRAF/ 
MEK/ERK inhibitors, cyclin-dependent kinase (CDK) inhibitors, PI3K/AKT/mTOR inhibitors]. The epigenetic inhibitors included enhancer of zeste homolog 2 (EZH2) inhibitors, histone deacetylase (HDAC) inhibitors, and isocitrate dehydrogenase (IDHs) inhibitors [28]. The use of such small molecules is not personalized; rather, it depends on the histological diagnosis of cancer and the characteristic biomarkers from cancer cells or blood from the patients [29]. Currently, 89 small molecule inhibitors have been approved by FDA and/or in China [28].

\section{Drugs targeting ferroptosis pathway}

Ferroptosis is a new approach in cancer therapy, which plays a critical role in minimizing the genesis process in the tumor by deleting the cells that lack nutrients in the surroundings. This necrosis is provoked by extra-mitochondrial lipid peroxidation arising from an iron-dependent reactive oxygen species accretion [30, 31]. Ferroptosis is involved in both tumor promotion as well as suppression. Anticancer therapy may target different pathways (epigenetic, transcriptional, post-transcriptional, or post-translational) that regulate the ferroptosis and stimulate the immune response within the tumor microenvironment. It has been tried in cancers, like breast cancer, melanoma, NSCLC, and pancreatic cancer [30]. Sorafenib, Lapatinib, BAY87-2243, and Lanperisone are the drugs that work through ferroptosis. Other than the clinically approved molecules, two antibiotics, salinomycin and ionomycin, promote ferroptosis and kill cancer cells, mainly in colon cancer [31]. Further, targeting ferroptosis may also help to revert drug resistance [32]. Drugs targeting ferroptosis may be combined with immunotherapy or radiotherapy to improve the efficacy [30].

\section{Stem cell-based therapy}

Cancer stem cells (CSCs) have been recently known for their role in the diagnosis, progression, and treatment of cancer. Although the interventional approaches in cancer targeting CSCs are relatively in the nascent stage, it is promising for long-term management and overcoming the challenges posed by conventional approaches (chemotherapy, radiotherapy, or immunotherapy) [33]. Cancer stem cells possess significant transformative properties, similar to any other stem cells and may be responsible for relapse, metastasis, or drug resistance. The self-renewal and transforming ability of CSCs is regulated by several intracellular and extracellular factors that could be targeted to treat cancer. A number of such surface or intracellular biomarkers of CSCs have been identified in different cancers. Besides, CSCs are controlled by several signaling pathways, major ones being Wnt, Notch,
Hedgehog, JAK-STAT, PI3K, and NF- $\kappa \beta$ signaling pathways $[33,34]$. Stem cells are also employed to develop anticancer vaccines, which are considered promising due to their high immunogenicity. Further genetic modification of CSCs can lend them useful as therapeutic carriers. This system (CSCs as therapeutic carriers) may be beneficial in preventing the degradation of therapeutic agents, increasing the anti-tumor effect of the agents, and reducing the adverse effects [33, 35].

\section{Nanotechnology in cancer therapy}

Nanotechnology has revolutionized cancer therapy by its numerous applications. The most useful application of nanotechnology has been tumor targeting by nanosized therapeutic agents. This targeting can be achieved by active (localization of nanoparticle-loaded drugs to specific antigens) or passive (increased permeability and retention) targeting [36]. The nanocarrier systems may include polymeric nanoparticles, polymer-drug nanoconjugates, liposomes, dendrimers, and inorganic nanoparticles. Nanoparticles can get loaded through different mechanisms, which involve non-covalent interaction, covalent interaction, and molecular interactions [37]. There are ample nano-formulations that have successfully entered the market, like nanocrystals, liposomes, lipidbased, polymeric, protein based, and metallic nanopharmaceuticals [38].

Using nanoparticles as the carrier also reduces the risk of early clearance by the reticuloendothelial system and provides sufficient time for accumulation in the tumor tissues. Further, these nanoparticles, once inside the tumor tissue, can produce cytotoxic reactions. However, the advantage of using nanoparticles over conventional methods is their specificity for the malignant cells (specific for molecular antigens expressed), sparing the host cells. The nanocarriers also help to increase the regression of the tumor cells while limiting the toxicity to normal cells [36].

Nanomedicines are also associated with challenges such as drugs' inability to be distributed to a specific destination and drug resistance that can render it ineffective. Although the associated benefits of nanoparticles are enormous, there are many limitations with nanoparticles, such as (a) difficulty in penetration due to the depth of tumor tissues, (b) clinical applicability as the nanoparticles are loaded with various agents, and (c) toxicity issues in patients [37].

Furthermore, nanotechnology can target multimodal approaches for cancer treatment [36]. Nanotechnology can also complement the implication of immunotherapy as it can increase its safety and efficacy $[39,40]$.

Table 1 describes the novel cancel therapy approaches which are at different stages of their journey. 
Table 1 Various novel therapy approaches

\begin{tabular}{|c|c|c|c|}
\hline Therapy approach & Type of cancer & $\begin{array}{l}\text { Clinical trial status of the approach (Yes } \\
\text { or No) }\end{array}$ & References \\
\hline Hormone Therapy & Metastatic cancers (breast or prostate) & Yes & Fairchild et al. [10] \\
\hline Cancer Immunotherapy & $\begin{array}{l}\text { Renal cancer, lung cancer, bladder } \\
\text { cancer, breast cancer, melanoma, } \\
\text { Hodgkin's lymphoma }\end{array}$ & Yes & Lee Ventola [13] \\
\hline Small-Molecule Inhibitors & Lymphomas & Yes, few have been approved too & Zhong et al. [28] \\
\hline Drugs Targeting Ferroptosis Pathway & $\begin{array}{l}\text { Like breast cancer, melanoma, NSCLC, } \\
\text { and pancreatic cancer }\end{array}$ & Yes & Chen et al. [30] \\
\hline Stem Cell-based Therapy & Blood cancer & Yes & Hayat et al. [33] \\
\hline Nanotechnology in Cancer Therapy & Neoplasms & Yes, few have entered in the market too & Farjadian et al. [38] \\
\hline
\end{tabular}

\section{Regulatory challenges associated with the development of novel cancer therapies}

Several challenges are posed in the way to the development of cancer therapies during the clinical trials, including clinical and regulatory challenges. The numerous clinical challenges posed are of concern. However, the research community addresses them well as we witness the improvement in understanding tumor biology and the targets for effective intervention. The scope of this article is confined to the regulatory challenges faced during the development process that hinders the availability of novel and effective therapies to a broader population.

Every nation has its regulatory framework and agencies to approve potential drugs for their clinical use. Each country in the European Union has its own regulatory agency. However, the applications for the oncology products are submitted by a centralized procedure in the European Union through European Medicines Agency (EMEA) $[24,41]$. The Food and Drug Administration (FDA) takes care of the drug approvals in the United States (US), while the Central Drugs Standard Control Organization (CDSCO) is the regulatory agency in India. Usually, a drug is approved for clinical use (marketing authorization) once the regulators are convinced that the therapeutic benefits outweigh the risks associated. The regulatory approval process typically involves a battery of preclinical evaluations, followed by fully controlled and well-defined clinical studies. The sample size and the study duration constitute prime importance for the regulatory agencies to decide for approval. However, this stringent procedure could not be applied for oncology products as it would pose a series of issues. Thus, the regulatory agencies have started to adopt a more flexible approach for the regulatory approval of cancer products. In addition to the customary approvals, other fast-track approval has also been adopted by regulatory agencies, like conditional approval by EMEA and accelerated approval by FDA [41].
The introduction of targeted and biological therapies in cancer has demanded flexibility in the regulatory process as new challenges emerged. Additionally, the concept of personalized therapy pile-up the challenges. The usual notion "one size does not fit all" is appropriate for defining oncology products' regulatory issues [42]. These challenges tend to increase the talks between industry and regulators and adopt a more flexible approach for anticancer products. Some of the challenges faced during the development of oncology medicines and their possible solutions are discussed in Table 2.

As discussed in the previous sections above, several cancer immunotherapeutic products are being developed, the most common and promising ones being mAbs. Both EMEA and FDA have their regulatory guidelines describing the development and approval process of mAbs in anticancer therapy. The regulation of the drugs targeting immunotherapy by the FDA falls under the Center for Biologics Evaluation and Research (CBER) or Center for Drug Evaluation and Research (CDER). CBER regulates the mAbs and proteins used for therapy, while CBER regulates the vaccines and blood products. CBER also regulates cancer vaccines as well as cell-based immunotherapy products. The guidance document of FDA, "Points to consider in the manufacture and testing of monoclonal antibody products for human use," released in 1997, is followed for the production and regulation of mAbs. Several amendments have been published later; however, this document remains the primary reference for the development of $\mathrm{mAbs}$ for cancer therapy ("Points to Consider in the Manufacture and Testing of Monoclonal Antibody Products for Human Use [43]. U.S. Food and Drug Administration Center for Biologics Evaluation and Research," 1997). Further, FDA has also passed the "Advanced Breakthrough Medicines for Patients" act and the "21 st Century Cures" act to focus on patient-directed development and increase the cross-talk between the developers and regulators. These amendments have helped to improve flexibility and speed for the approval and even considered the role of biomarkers [41]. 


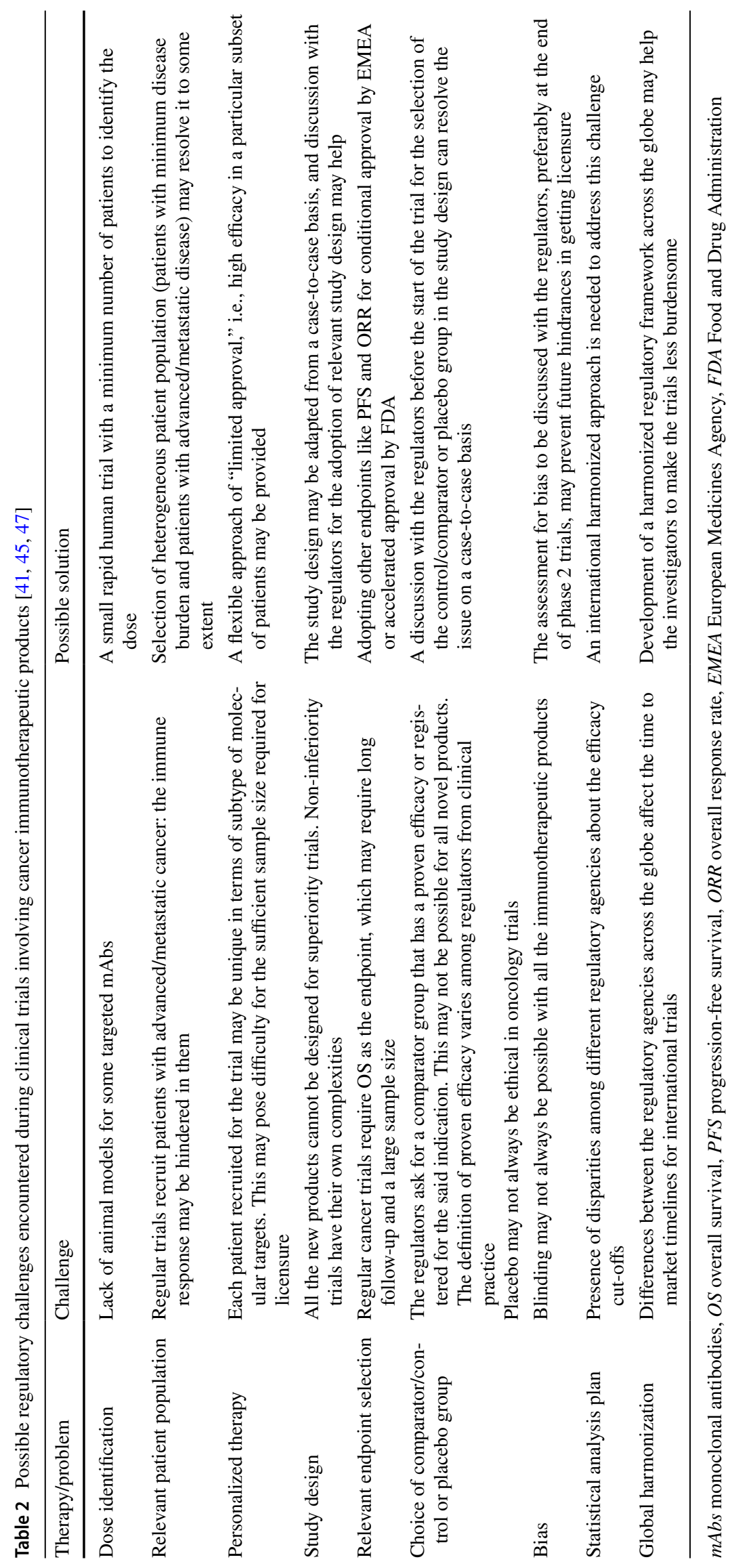


EMEA released its 5th revision of "Guideline on the evaluation of anticancer medicinal products in man" in September 2017 that explained the development of anticancer products, including mAbs, throughout their clinical journey of all the phases. This revision has included the concept of a biomarker specific for the cancer type related to the treatment. The guidelines ask for biomarker investigations in addition to the documents showing the efficacy and safety of the treatment. The biomarkers can serve as "proof of concept," a tool for deciding the dose, identifying the eligible patients, and resistance mechanisms. It can also help to determine the efficacy and safety of the therapy [44]. Thus, the regulatory guidelines have now included the concept of biomarkers to better evaluate the risk-to-benefit ratio for a particular therapy.

\section{Cancer immunotherapeutic products}

One of the prominent challenges posed by immunotherapeutic products is demonstrating the efficacy in specific cancer patients. The difficulty faced includes the sample size, choice of primary endpoints in advanced cancer patients enrolled in clinical trials, lack of comparator or placebo-controlled groups for demonstrating comparable efficacy of new cancer products, and the duration of the therapy in advanced cancer patients $[41,45,46]$. Besides, early clinical trials designed to establish the safety of the new products also encounter specific difficulties. The starting dose for first in human studies may not be available for some mAbs from the preclinical studies, as they lack a relevant animal model for the specific antigen. This challenge may be handled by designing a small human trial with a minimum number of subjects to identify the dose rapidly. Alternatively, other methods like accelerated titration or continuous reassessment may also be employed instead of the standard methods [47].

The selection of the relevant patient population is a significant challenge for most of the new immunotherapeutic products. Most of the studies for the chemotherapeutic agents involve patients with advanced or metastatic cancer. These agents are tried in patients with the minimal disease only after the effectiveness and safety are confirmed. Such patients may show the effectiveness of the agent much rapidly as the disease progression is fast in them. The disease progression may not be fast with some immunotherapeutic agents like cancer vaccines and require a longer time to develop a sufficient immune response. Further, the immune system may be hindered in metastatic patients as they may have encountered previous immunosuppressive therapies, like chemo or radiotherapies. Therapies like cancer vaccines may not be able to produce a significant immune response in such patients. On the other hand, testing cancer vaccines in patients with early disease would require a strict follow-up, possible with only randomized trials. Thus, selecting a patient population for testing cancer vaccines for licensure would be challenging. The regulators allow for a heterogeneous patient population for such trials; however, proper consideration must be given for selecting a heterogeneous patient population [47].

Immunotherapies are usually targeted therapies, and the biomarker-response relationship is very complex. Each cancer patient may possess or evolve to possess more than one subtype of the molecular targets during their tumor development. This may impede the development of new immunotherapeutic products as the responding behavior of all the patients enrolled in the study may not be similar. Further, the disease control may be observed up to a particular stage of the disease, after which other targeted therapy may be needed to control the disease. This uniqueness of each cancer patient may pose a significant challenge in enrolling enough patients (i.e., around 100-150) among the subset required for either accelerated or regular approvals. This challenge could be won by accepting a flexible approach of "limited approval" criteria suggested by [41]. This approval would be based on the high efficacy without significant nonmechanism-related adverse effects among a particular subset of the target population (maybe small) and the presence of biomarkers may support that. This approval may not be the end, and the research work may continue to add the survival data and identify better biomarkers. Such an approval process may be helpful for a limited, stringently defined group of patients [41].

The increase in the overall survival (OS, time from enrollment to death) was considered as the most relevant endpoint in all the oncology trials. However, the pre-requisites for this endpoint were a large sample size and a sufficiently long time for follow-up. Further, it is also unethical and not feasible for the control patients enrolled in the study. They may opt for some more effective therapy or switch the groups or even withdraw from the study [45]. This challenge is now addressed by adopting progression-free survival (PFS, time from enrollment to tumor progression) as the preferred primary endpoint by European Union [44, 45]. In contrast, FDA still considers PFS as a surrogate marker to OS for some cancers while as a measure of clinical benefit for other cancers, such as NSCLC, colorectal cancer, and ovary cancer $[45,48]$. Nonetheless, the challenge of defining and identifying the tumor progression still exists.

The choice of the comparator or placebo group is another challenge posed in the way of regulatory approval. The agent chosen to be the comparator/control group should be either proven efficacious or standard care therapy. However, the definition of proven efficacy for many regulators may differ from the usual clinical practice and maybe a hurdle in superiority trials seeking regulatory approval. The comparator/ control drug should ideally be a registered therapy for the 
said indication of the trial, which may not always be the case. In such situations, the designation of the non-investigational product may not be rendered to the control medication, thus, leading to several logistic and financial issues. A possible solution to this issue would be to arrange for advice from the regulator for the comparator product before the start of the trial [45].

Further, the use of placebo-controlled trials is another ethical and regulatory problem in oncology trials. The cancer trials wherein therapies improving the survival or quality of life are pre-existing, placebo groups with no treatments are usually avoided as they are unethical and thus not accepted for regulatory approvals. Further, FDA allows for single-arm trial designs for cancers that are rare or with no pre-existing therapies to resolve the use of placebos [46, 47].

The study design of the immunotherapeutic product may also be challenging as the product's efficacy, and safety must be proven compared to the pre-existing therapy for a particular condition. These trials can be designed as superiority trials; however, they may require a large sample size. Thus, non-inferiority trials are preferred or the study design may be adapted on a case-to-case basis. FDA provides guidance for designing adaptive trials "Adaptive Designs for Clinical Trials of Drugs and Biologics-Guidance for Industry" in November 2019 that may be referred for overcoming the challenge of complex study designs [47, 49].

Usually, blinding helps to remove the bias in clinical trials. However, certain trials (e.g., cancer vaccines) could be easily detected owing to the reactions by the therapeutic agents or co-administered cytokines. Thus, to maintain the blinding status, the person monitoring each stage should be separate (drug administration, patient care after administration, or endpoint evaluation). Besides, other agents like mAbs may also produce adverse effects, which may hinder the blinding process. FDA suggests the assessment of bias to be conducted at the end of phase 2 trials and discussed with the regulators to ensure that no bias exists in the study $[45,50]$.

Another significant challenge is in the statistical analysis plan. Different regulatory agencies offer different statistical cut-offs for the trial to be considered positive or negative. Thus, the same trial may be regarded as positive in one regulatory region, while negative in another region. This disparity has propped a need for an internationally harmonized approach for statistical analysis plan after consulting all the major regulatory agencies of the globe [45].

Last but not the least is the challenge of global harmonization of the regulations pertaining to oncology trials. It has been discussed at various forums like the American Society of Clinical Oncology workshop. The difference in the regulations for conditional approval by the EU and accelerated approval by FDA were addressed in this forum. FDA grants accelerated approval based on the efficacy of surrogate markers showing clinical benefit, while the EU may grant conditional approval if the benefit-risk ratio of the therapy is positive. Both the regulators require post-marketing studies to be carried for proving the benefit. The FDA suggests improvement in OS while the EU asks for a positive benefit-risk ratio [50]. The approval of bevacizumab was an example that was revoked by the FDA as it could not show improvement in OS in breast cancer while it got approval by EMEA as the benefit-to-risk ratio was shown positive for the combination of bevacizumab with paclitaxel [51, 52]. Such disparities between the regulatory agencies may affect the time to market approval for the international trials. All the regulatory agencies of different countries work on similar principles; however, they differ in their scope and documents to be submitted. Thus, developing a harmonized regulatory framework across the globe may help the investigators make the trials less burdensome [53, 54].

\section{Cancer vaccines}

The endpoint selection can be critical for cancer vaccines. They may be more efficacious for improving OS rather than PFS or radiological improvement in the tumor. Thus, patients should not be dropped from the study based on the progression of the disease. This issue can be resolved by defining all the situations in the study protocol clearly [47]. Also, patient-reported outcomes may be of significance to support licensure as a major clinical benefit, described in "Guidance for Industry: Patient-Reported Outcome Measures: Use in Medical Product Development to Support Labeling Claims," published in December 2009 by FDA. FDA has also published a guidance document, "Clinical considerations for Therapeutic Cancer Vaccines," for the design of clinical trials of cancer vaccines [55]. In addition, sometimes adjuvants (e.g., cytokines) are used along with cancer vaccines to increase the immune response against the specified antigen. The regulatory agencies require complete details of the adjuvants at an early stage of vaccine development, especially if the adjuvants have independent clinical activity. A one-on-one discussion with the regulators can help develop the study design in these cases [47].

\section{Cell-based immunotherapeutic products}

Even though CAR-T-cell products (e.g., CD19-targeted B-cell acute lymphocytic leukemia) have been given breakthrough therapy designation, but that does not provide the opportunity to get the Biological License Application (BLA) review on a priority basis. Its approval on an acceleration basis was dependent upon surrogate endpoints in pivotal trials. Further, the complete approvals were provided based on the post-marketing studies for CAR-T-cell therapies. As there are associated toxicities with CAR-T therapy, the 
FDA demanded for Risk Evaluation and Mitigation Strategy (REMS) to be submitted by manufacturers to ensure longterm safety [56]. Currently, two CAR-T-cell therapies are approved, Kymriah and Yescarta, by FDA and EMEA. This therapy is challenging for regulatory authorities to classify it as a drug, device, or hybrid. Further, the challenges are pronounced for this therapy due to difficulties faced during quality control as well as the absence of long-term efficacy and safety data [57].

\section{Stem cell-based therapy}

The stem cell marketing business is highly popular in developed nations like the US, Japan, and Australia. The direct marketing of stem cells to consumers is highly growing for therapeutic uses that are not proven. This strategy of selling these stem cells is becoming highly beneficial for the sellers. However, there are various limitations and gaps associated with clinical studies of stem cells. The concept of "pay to participate" brings a challenge because there are various ethical concerns linked with such clinical studies. This may unnecessarily bring the subjects into the situation of getting harmed. Also, all the studies are not registered, and all registered on the National Institutes of Health (NIH) are not under FDA supervision. The loopholes with regulatory guidelines push sellers to promote such products as "personalized" medicines. The patient-funded research provides insulation from litigation to the providers. The US has developed a "dual" system wherein the companies are required to get engaged with FDA and pass-through strict pre-market testing through various phases of clinical trials to generate enough results to prove the safety and efficacy of the product. The FDA is trying to minimize the loopholes; however, it will be taking time to make guidelines streamlined [58].

On comparing the regulatory developments in seven countries, like Argentina, Brazil, China, the EU, India, Japan, and the USA, it was concluded that challenges for international clinical trial collaborations emerge due to the variations in the review process of regulatory authorities. The list of these challenges includes high costs, delays in reaching medical progress, enhanced administrative issues, and the requirement of scientific self-governance. These challenges delay the patient's access to stem cell therapies with clinically proven status [59].

In India, it has been observed that the clinics provide stem cell therapies, lacking proven therapeutic efficacy for the given indication. Along with developed nations, developing nations are also using the same strategy to gain benefits out of these therapies without proving the clinical efficacy of these therapies. This misuse is mainly due to a huge gap in regulations and a lack of policies. However, The Indian Council of Medical Research (ICMR) has raised a voice to place "stem cells" into drugs [60].

\section{Nanotechnology-based therapy}

In the current era, nanocarriers are also taking a vast place and are used as targeted therapy in cancer. Despite being numerous successful preclinical studies happening, there are just 15 targeted nanocarriers for clinical trials. Since much of the preclinical research for nanocarriers is conducted by academia, compliance to good laboratory practices may be lacking, questioning the validity and reproducibility of scientific results. This could further create a gap regarding collaboration with the pharmaceutical industry. Thus, there is a considerable gap in commercialization, leading to issues in regulatory approvals [61]. The US FDA has recently approved several nanomedicinebased cancer vaccines; however, many are under the clinical evaluation and translation stage. One of the pivotal challenges for the regulatory authorities to approve a nanobased therapy is its safety [62].

Several challenges that are discussed above for immunotherapeutic agents also apply to nano-based therapy approaches. The evaluation of its safety and efficacy, a pre-requisite for any regulatory approval, is complicated due to nanocarriers' complex structure and chemical composition. Also, placebo-controlled trials may not be feasible for such therapies and thus alternatives need to be explored. The selection of the patient population, as well as the study design, is very crucial. The target receptor presence, tumor heterogeneity, binding of nanocarrier with the target receptor, and supportive diagnostics need to be considered in the study design and patient selection [61]. Besides, certain issues demand immediate action from regulatory authorities like preclinical in vivo tests and the economic impact of the clinical trials on the total cost. An extension in the period of market exclusivity for such products may help the developers to avail the maximum profit of the product [38].

\section{Conclusion}

Cancer therapy has been revolutionized with the introduction of novel targeted therapies, like immunotherapy, cancer vaccines, stem cell-based therapies, or nanocarrierbased therapies. These therapies have addressed several issues related to conventional therapy (chemotherapy and radiotherapy), like specific cytotoxic action toward malignant cells, side effects, and overall survival. However, these therapies face several clinical, ethical, and regulatory challenges at each step of their development. The regulatory concerns are the major ones that pause the introduction of effective therapies in the market. Further, 
the difference in the regulations across the globe makes the process burdensome and delays the time to market. Thus, it is high time for the regulatory authorities across the world to work together to create a robust framework for new targeted therapies and ease their entry into the market without compromising on the efficacy and safety of the therapies.

Funding There was no funding for writing this review.

\section{Declarations}

Conflict of interest Pinky Sharma, Pooja Mathur, and Rohit Dutt declare that they have no conflict of interest.

Research involving human and animal rights This article does not contain any studies with human participants or animals performed by any of the authors.

\section{References}

1. Sung H, Ferlay J, Siegel RL, Laversanne M, Soerjomataram I, Jemal A, Bray F. Global Cancer Statistics 2020: GLOBOCAN estimates of incidence and mortality worldwide for 36 cancers in 185 countries. A Cancer J Clin. 2021;71(3):209-49. https://doi. org/10.3322/caac. 21660 .

2. https://www.uicc.org/news/globocan-2020-new-global-cancerdata (2020). Accessed 2 Aug 2021.

3. Smith RD, Mallath MK. History of the growing burden of cancer in India: From antiquity to the 21 st century. J Glob Oncol. 2019;2019(5):1-15. https://doi.org/10.1200/JGO.19.00048.

4. Baudino T. Targeted cancer therapy: the next generation of cancer treatment. Curr Drug Discov Technol. 2015;12(1):3-20. https:// doi.org/10.2174/1570163812666150602144310.

5. Falzone L, Salomone S, Libra M. Evolution of cancer pharmacological treatments at the turn of the third millennium. Front Pharmacol. 2018;9:1300. https://doi.org/10.3389/fphar.2018.01300.

6. Aquib M, Farooq MA, Filli MS, Boakye-Yiadom KO, Kesse S, Maviah MBJ, Mavlyanova R, Wang B. A review on the chemotherapeutic role of fucoidan in cancer as nanomedicine. Rjlbpcs. 2019;5(1):512-39. https://doi.org/10.26479/2019.0501.44.

7. Shewach DS, Kuchta RD. Introduction to cancer chemotherapeutics. Chem Rev. 2009;109(7):2859-61. https://doi.org/10.1021/ cr900208x.

8. Schirrmacher V. From chemotherapy to biological therapy: a review of novel concepts to reduce the side effects of systemic cancer treatment (review). Int J Oncol. 2019;54(2):407-19. https://doi.org/10.3892/ijo.2018.4661.

9. Zugazagoitia J, Guedes C, Ponce S, Ferrer I, Molina-Pinelo S, Paz-Ares L. Current challenges in cancer treatment. Clin Ther. 2016;38(7):1551-66. https://doi.org/10.1016/j.clinthera.2016.03. 026.

10. Fairchild A, Tirumani SH, Rosenthal MH, Howard SA, Krajewski KM, Nishino M, Shinagare AB, Jagannathan JP, Ramaiya NH. Hormonal therapy in oncology: a primer for the radiologist. Am J Roentgenol. 2015;204(6):W620-30. https://doi.org/10.2214/AJR. 14.13604.

11. Abraham J, Staffurth J. Hormonal therapy for cancer. Medicine. 2016;44(1):30-3. https://doi.org/10.1016/j.mpmed.2015.10.014.
12. Pucci C, Martinelli C, Ciofani G. Innovative approaches for cancer treatment: current perspectives and new challenges. Ecancermedicalscience. 2019;13:1-26. https://doi.org/10.3332/ecanc er.2019.961.

13. Lee Ventola C. Cancer immunotherapy, Part 1: current strategies and agents. Pharm Ther. 2017;42(6):375-83.

14. Lee Ventola C. Cancer immunotherapy, Part 2: efficacy, safety, and other clinical considerations. Pharm Ther. 2017;42(7):452-63.

15. Zahavi D, Weiner L. Monoclonal antibodies in cancer therapy. Antibodies. 2020;9(3):34. https://doi.org/10.3390/antib9030034.

16. Baldo BA. Safety of biologics therapy. Saf Biol Ther. 2016. https://doi.org/10.1007/978-3-319-30472-4.

17. Lu RM, Hwang YC, Liu IJ, Lee CC, Tsai HZ, Li HJ, Wu HC. Development of therapeutic antibodies for the treatment of diseases. J Biomed Sci. 2020;27(1):1-30. https://doi.org/10.1186/ s12929-019-0592-z.

18. Guo F, Cui J. Anti-angiogenesis: opening a new window for immunotherapy. Life Sci. 2020;258:118163. https://doi.org/10. 1016/j.lfs.2020.118163.

19. Fallah A, Heidari HR, Bradaran B, Sisakht MM, Zeinali S, Molavi O. A gene-based anti-angiogenesis therapy as a novel strategy for cancer treatment. Life Sci. 2019;239:117018. https://doi.org/10. 1016/j.lfs.2019.117018.

20. Jászai J, Schmidt MHH. Trends and challenges in tumor antiangiogenic therapies. Cells. 2019;8(9):7-11. https://doi.org/10. 3390/cells8091102.

21. Sambi M, Bagheri L, Szewczuk MR. Current challenges in cancer immunotherapy: multimodal approaches to improve efficacy and patient response rates. J Oncol. 2019. https://doi.org/10.1155/ 2019/4508794.

22. Waldman AD, Fritz JM, Lenardo MJ. A guide to cancer immunotherapy: from $\mathrm{T}$ cell basic science to clinical practice. Nat Rev Immunol. 2020;20(11):651-68. https://doi.org/10.1038/ s41577-020-0306-5.

23. Sterner RC, Sterner RM. CAR-T cell therapy: current limitations and potential strategies. Blood Cancer J. 2021;11(4):1-11. https:// doi.org/10.1038/s41408-021-00459-7.

24. Camarero J, Ruiz S. Cancer immunotherapy products. Hum Vaccin Immunother. 2012;8(9):1354-9. https://doi.org/10.4161/hv. 21142.

25. Hollingsworth RE, Jansen K. Turning the corner on therapeutic cancer vaccines. Npj Vaccines. 2019;4(1):1-10. https://doi.org/ 10.1038/s41541-019-0103-y.

26. Barbari C, Fontaine T, Parajuli P, Lamichhane N, Jakubski S, Lamichhane P, Deshmukh RR. Immunotherapies and combination strategies for immuno-oncology. Int J Mol Sci. 2020;21(14):1-28. https://doi.org/10.3390/ijms21145009.

27. Mokhtari RB, Homayouni TS, Baluch N, Morgatskaya E, Kumar $\mathrm{S}$, Das B, Yeger H. Combination therapy in combating cancer. Oncotarget. 2017;8(23):38022-43. https://doi.org/10.18632/oncot arget.16723.

28. Zhong L, Li Y, Xiong L, Wang W, Wu M, Yuan T, Yang W, Tian C, Miao Z, Wang T, Yang S. Small molecules in targeted cancer therapy: advances, challenges, and future perspectives. Signal Transduct Targeted Ther. 2021;6(1):1-48. https://doi.org/10.1038/ s41392-021-00572-w.

29. Bedard PL, Hyman DM, Davids MS, Siu LL. Small molecules, big impact: 20 years of targeted therapy in oncology. The Lancet. 2020;395(10229):1078-88. https://doi.org/10.1016/S01406736(20)30164-1.

30. Chen X, Kang R, Kroemer G, Tang D. Broadening horizons: the role of ferroptosis in cancer. Nat Rev Clin Oncol. 2021;18(5):280 96. https://doi.org/10.1038/s41571-020-00462-0.

31. Mou Y, Wang J, Wu J, He D, Zhang C, Duan C, Li B. Ferroptosis, a new form of cell death: opportunities and challenges in 
cancer. J Hematol Oncol. 2019;12(1):1-16. https://doi.org/10. 1186/s13045-019-0720-y.

32. Wu Y, Yu C, Luo M, Cen C, Qiu J, Zhang S, Hu K. Ferroptosis in cancer treatment: another way to Rome. Front Oncol. 2020;10:1924. https://doi.org/10.3389/fonc.2020.571127.

33. Hayat H, Hayat H, Francis B, Gudi DM, Bishop JO, Wang P. A concise review: the role of stem cells in cancer progression and therapy. Onco Targets Ther. 2021;14:2761-72. https://doi.org/10. 2147/OTT.S260391.

34. Yang L, Shi P, Zhao G, Xu J, Peng W, Zhang J, Zhang G, Wang X, Dong Z, Chen F, Cui H. Targeting cancer stem cell pathways for cancer therapy. Signal Transduct Targeted Ther. 2020;5(1):1-35. https://doi.org/10.1038/s41392-020-0110-5.

35. Chu DT, Nguyen TT, Tien NLB, Tran DK, Jeong JH, Anh PG, Thanh VV, Truong DT, Dinh TC. Recent progress of stem cell therapy in cancer treatment: molecular mechanisms and potential applications. Cells. 2020;9(3):1-19. https://doi.org/10.3390/cells 9030563.

36. Gmeiner WH, Ghosh S. Nanotechnology for cancer treatment. Nanotechnol Rev. 2014;3(2):111-22. https://doi.org/10.1515/ ntrev-2013-0013.

37. Zhao CY, Cheng R, Yang Z, Tian ZM. Nanotechnology for cancer therapy based on chemotherapy. Molecules. 2018;23(4):826. https://doi.org/10.3390/molecules23040826.

38. Farjadian F, Ghasemi A, Gohari O, Roointan A, Karimi M, Hamblin MR. Nanopharmaceuticals and nanomedicines currently on the market: challenges and opportunities. Nanomedicine. 2019;14(1):93-126. https://doi.org/10.2217/nnm-2018-0120.

39. Goldberg MS. Improving cancer immunotherapy through nanotechnology. Nat Rev Cancer. 2019;19(10):587-602. https://doi. org/10.1038/s41568-019-0186-9.

40. van der Meel R, Sulheim E, Shi Y, Kiessling F, Mulder WJM, Lammers T. Smart cancer nanomedicine. Nat Nanotechnol. 2019;14(11):1007-17. https://doi.org/10.1038/ s41565-019-0567-y.

41. Dhingra K. Oncology 2020: a drug development and approval paradigm. Ann Oncol. 2015;26(11):2347-50. https://doi.org/10. 1093/annonc/mdv353.

42. An American Society of Clinical Oncology and Institute of Medicine Workshop; Institute of Medicine; National Cancer Policy Forum; Board on Health Care Services. Implementing a National Cancer Clinical Trials System for the 21st Century: Second Workshop Summary. Washington (DC): National Academies Press (US); 2013 Sep 27. REGULATORY ISSUES. https://www.ncbi. nlm.nih.gov/books/NBK202100/

43. Points to consider in the manufacture and testing of monoclonal antibody products for human use (1997). U.S. Food and Drug Administration Center for Biologics Evaluation and Research. J Immunother. 1997; 20(3), 214-243. https://doi.org/10.1097/00002 371-199705000-00007

44. Agency EM. Committee for Medicinal Products for Human Use (CHMP) - Guideline on the evaluation of anticancer medicinal products in man. Eur Med Agency. 2017;44:1-43.

45. Augustus $\mathrm{S}$. Drug development in oncology: a regulatory perspective. Am J Ther. 2011;18(4):323-31. https://doi.org/10.1097/MJT. 0b013e3181d1d833.

46. Daugherty CK, Ratain MJ, Emanuel EJ, Farrell AT, Schilsky RL. Ethical, scientific, and regulatory perspectives regarding the use of placebos in cancer clinical trials. J Clin Oncol. 2008;26(8):13718. https://doi.org/10.1200/JCO.2007.13.5335.

47. Vatsan RS, Bross PF, Liu K, Theoret M, De Claro AR, Lu J, Helms W, Niland B, Husain SR, Puri RK. Regulation of immunotherapeutic products for cancer and FDA's role in product development and clinical evaluation. J Immunother Cancer. 2013;1:116. https://doi.org/10.1186/2051-1426-1-5.
48. Research, C. for D. E. https://www.fda.gov/media/71195/downl oadClinicaltrial endpoints for the approval of cancer drugs and biologics: guidance for industry. U.S. Food and Drug Administration; 2018, p. 1-16.

49. FDA. 2019. Adaptive Design Clinical Trials for Drugs and Biologics Guidance for Industry. https://www.fda.gov/regulatory-infor mation/search-fda-guidance-documents/adaptive-design-clinicaltrials-drugs-and-biologics-guidance-industry

50. Medicine, I. of. Implementing a National Cancer Clinical Trials System for the 21st Century: Second Workshop Summary Nass, S.J., Patlak, M. editors. The National Academies Press; 2013. https://doi.org/10.17226/18362

51. Benstetter M, Haubenreisser S. European Medicines Agency completes its review of Avastin used in breast cancer treatment EMA/ CHMP. 44. http://www.ema.europa.eu/docs/en_GB/document library/Press_release/2010/12/WC500099929.pdf

52. Burstein HJ. Bevacizumab for advanced breast cancer: all tied up with a RIBBON? J Clin Oncol. 2011;29(10):1232-5. https://doi. org/10.1200/jco.2010.33.2684.

53. Salcher-Konrad M, Naci H, Davis C. Approval of cancer drugs with uncertain therapeutic value: a comparison of regulatory decisions in Europe and the United States. Milbank Q. 2020;98(4):1219-56. https://doi.org/10.1111/1468-0009.12476.

54. Tang M, Joensuu H, Simes RJ, Price TJ, Yip S, Hague W, Sjoquist KM, Zalcberg J. Challenges of international oncology trial collaboration — a call to action. Br J Cancer. 2019;121(7):515-21. https://doi.org/10.1038/s41416-019-0532-4.

55. Vaccines TC. Guidance for industry clinical considerations for therapeutic cancer vaccines [excerpts]. Biotechnol Law Rep. 2012;31(3):303-9. https://doi.org/10.1089/blr.2012.9874.

56. Locke FL, Davila ML. Regulatory challenges and considerations for the clinical application of CAR-T cell anti-cancer therapy. Expert Opin Biol Ther. 2017;17(6):659-61. https://doi.org/10. 1080/14712598.2017.1322953.

57. Ou Z. Pcn 198 global regulatory challenges of car T-cell therapies: approval, pricing, and access. Value Health. 2019;22(June):S93. https://doi.org/10.1016/j.jval.2019.04.320.

58. Sipp D. Challenges in the regulation of autologous stem cell interventions in the United States. Perspect Biol Med. 2018;61(1):2541. https://doi.org/10.1353/pbm.2018.0025.

59. Rosemann A, Bortz G, Vasen F, Sleeboom-Faulkner M. Global regulatory developments for clinical stem cell research: diversification and challenges to collaborations. Regen Med. 2016;11(7):647-57. https://doi.org/10.2217/rme-2016-0072.

60. Tiwari SS, Desai PN. Unproven stem cell therapies in India: regulatory challenges and proposed paths forward. Cell Stem Cell. 2018;23(5):649-52. https://doi.org/10.1016/j.stem.2018.10.007.

61. Rosenblum D, Joshi N, Tao W, Karp JM, Peer D. Progress and challenges towards targeted delivery of cancer therapeutics. Nat Commun. 2018;9(1):1-12. https://doi.org/10.1038/ s41467-018-03705-y.

62. Beg S, Alharbi KS, Alruwaili NK, Alotaibi NH, Almalki WH, Alenezi SK, Altowayan WM, Alshammari MS, Rahman M. Nanotherapeutic systems for delivering cancer vaccines: recent advances. Nanomedicine. 2020;15(15):1527-37. https://doi.org/ 10.2217/nnm-2020-0046.

Publisher's Note Springer Nature remains neutral with regard to jurisdictional claims in published maps and institutional affiliations. 